\title{
Búsqueda activa de casos de sobrecarga de hierro en un hospital universitario
}

\author{
P. KHOSRAVI SHAHI, M. CAÑO HORTONEDA, A. DEL CASTILLO RUEDA, J. DE \\ PORTUGAL ÁLVAREZ
}

Unidad de Ferropatología y Radicalosis. Servicio de Medicina Interna II. Hospital General Universitario Gregorio Marañón. Madrid

CASE FINDING OF IRON OVERLOAD IN A UNIVERSITY HOSPITAL

\section{RESUMEN}

Fundamento: La importancia epidemiológica de las enfermedades por depósito de hierro y la insuficiencia clínica en su diagnóstico nos lleva a realizar una búsqueda activa en pacientes ingresados en un hospital de tercer nivel lo que permitirá un aumento en la detección de casos nuevos y una intervención preventiva y terapéutica eficaz.

Pacientes y métodos: Se realizó un estudio transversal de 4 meses de duración de los pacientes que ingresaron en una sección de quince camas de medicina interna de un hospital general universitario. A todos ellos, sin criterios de exclusión, se les realizó el estudio de los parámetros del metabolismo del hierro. El índice de saturación de transferrina (IST) por su sensibilidad, especificidad y valor predictivo positivo es el primero a efectuar para el cribado fenotípico de las enfermedades de sobrecarga de hierro considerándose el umbral de sospecha (población de alto riesgo o cribado positivo) cuando es igual o superior a $45 \%$.

Resultados: El tamaño muestral fue de 117 pacientes con una edad media

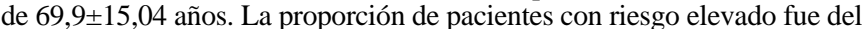
$13,68 \%$ ( 16 casos), con una edad media de 60,8 20,17 años, frente a la población de bajo riesgo (101 casos) que tenía una media de edad de 71,3ะ13,7 años $(\mathrm{p}<0,05)$. Las enfermedades más frecuentemente asociadas a la población de alto riesgo son diabetes mellitus y hepatopatía crónica $(31,25 \%)$. El $18,75 \%$ de los casos positivos ( 3 de 16) presentaban desnutrición.

Conclusiones: La detección de casos nuevos de sobrecarga de hierro se puede impulsar mediante la búsqueda activa en pacientes ingresados a los que se realiza el IST que resulta una técnica de cribado de alta rentabilidad diagnóstica, valorando adecuadamente procesos asociados y falsos positivos. Por todo ello, creemos necesaria la inclusión del IST dentro de las peticiones analíticas a realizar en todos los pacientes a su ingreso.

PALABRAS CLAVE: Sobrecarga de hierro. Hemocromatosis. Índice de saturación de transferrina. Cribado.

\section{ABSTRACT}

Background: The epidemiologic importance of iron overload disor ders and the clinical lack of its diagnosis led us to an active search of cases among the hospitalized patients in a general university hospital. This situation will make it possible to have a larger and earlier number of diagnosis together with a preventive intervention and an effective tre atment.

Patients and methods: A transverse study was performed on patients admitted to a fifteen bedsward in the internal medicine department. They have all been applied, without exclusion criteria, the iron metabo lism parameters survey. The transferrin saturation (TS), due to its sensi tivity, specificity and positive predictive value, has been considered appropriate for the phenotypic screening of the iron overload disorders. Subjects with fasting TS of 45\% or more were considered as risk popu lation.

Results: The study comprised 117 patients with an average age of 69.9 15.04 . The high-risk population rate was $13.8 \%$ with an average age of $60.8 \pm 20.17$, as compared to the low-risk population with an average age of $71.3 \pm 13.7(p<0.05)$. The most outstanding illnesses associa ted to the high-risk population are chronic hepatitis and diabetes melli tus $(31.25 \%)$. An $18.7 \%$ of the cases show undernourishment.

Conclusions: The iron overload is a prevalent illness whose early diagnosis requires a high clinical suspicion. The active search among the hospitalized patients, who have been made a TS, benefits the new cases detection. The TS is a screening technique with a high diagnostic profitability if you evaluate correctly the false positives. For that reason, we think the TS has to be included within the analytic requests which patients are made when admitted to hospital.

KEY WORDS: Iron overload. Hemochromatosis. Transferrin satura tion. Screening.

Khosravi Shahi P, Caño Hortoneda M, Del Castillo Rueda A, De Portugal Álvarez. J. Búsqueda activa de casos de sobrecarga de hierro en un hospital universitario. An Med Interna (Madrid) 2003; 20: 67-69.

\section{INTRODUCCIÓN}

Las enfermedades de sobrecarga de hierro (ESF) incluyen las formas hereditarias o primarias y las secundarias o adquiri- das (1). La hemocromatosis hereditaria (HH) es una enfermedad metabólica que se caracteriza por sobrecarga y depósito de hierro en las células parenquimatosas de diferentes órganos, fundamentalmente hígado, corazón y páncreas, inducien-

Trabajo aceptado: 25 de octubre de 2002

Correspondencia: Alejandro del Castillo Rueda. Servicio de Medicina Interna II. Hospital General Universitario Gregorio Marañón. Doctor Esquerdo, 46. 28007 Madrid. 
do su deterioro funcional y estructural (2). Se considera la enfermedad genética más frecuente en la población humana de origen caucasiano $(3,4)$.

Por sus características clínicas, la hemocromatosis es una enfermedad adecuada para la realización de programas de cribado poblacional: es prevalente (afecta al menos a 5 de cada 1.000 ciudadanos), potencialmente grave, tiene un largo periodo asintomático, se dispone de pruebas para confirmar el diagnóstico y se conoce un tratamiento eficaz si se realiza precozmente (5-7).

El estudio del metabolismo del hierro, que incluye sideremia, transferrina, índice de saturación de transferrina (IST) y ferritina, se utiliza como método de cribado fenotípico. El IST es el parámetro que se considera como la mejor técnica para el diagnóstico precoz de la enfermedad por su sensibilidad, especificidad y valor predictivo positivo $(4,8)$. La ferritina es un marcador de acúmulo de hierro y no es útil para el diagnóstico precoz cuando aún la enfermedad no ha evolucionado (9).

Con el fin de aumentar la detección de casos incidentes en fase inicial o asintomática, se han diseñado estrategias tanto en el ámbito hospitalario como en asistencia primaria (10). La búsqueda activa de casos en pacientes ingresados (11) consiste en realizar el cribado fenotípico para detectar los estados de sobrecarga de hierro y sus posibles enfermedades asociadas y poder establecer medidas preventivas y terapéuticas eficaces (5).

\section{PACIENTES Y MÉTODOS}

Estudio epidemiológico transversal (descriptivo), de 4 meses de duración (desde el 15 de enero al 15 de mayo de 2002), usando como población muestral los pacientes ingresados en una sección de 15 camas del servicio de medicina interna de un hospital general universitario. Durante este período se realizó el cribado fenotípico a todos los pacientes que ingresaron, sin establecer ningún criterio de exclusión.

El cribado fenotípico consiste en el estudio de los parámetros del metabolismo del hierro: sideremia, transferrina, índice de saturación de transferrina (IST) y ferritina, usando el IST en ayunas como el primer marcador de cribado. Se considera el umbral de sospecha de sobrecarga de hierro a partir de un IST de $45 \%$ que frente a valores de corte más elevados, optimiza la sensibilidad aunque la especificidad sea menor $(13,14)$. Así obtendremos una población dividida en dos grupos de riesgo:

-Cribado negativo o bajo riesgo (IST menor de $45 \%$ ). $45 \%)$.

- Cribado positivo o riesgo elevado (IST igual o mayor de

En todos los pacientes incluidos se recogían las características clínicas y epidemiológicas: sexo, edad, raza, antecedentes médicos, antecedentes quirúrgicos, tratamiento previos y el motivo de ingreso. Los pacientes con cribado positivo y sin causa conocida de la sobrecarga de hierro fueron enviados a consulta externa al alta hospitalaria para realización de estudio genético.

Para el análisis de los datos se ha aplicado el programa informático de estadísticas, denominado Editor de Datos SPSS.

\section{RESULTADOS}

El tamaño muestral fue de 117 pacientes, con una edad media de 69,9 años $\pm 15,04$ (mediana de 73 años y rango intercuartílico de 15 años). El 51,3\% eran varones y 48,7\% mujeres, según la tabla I.
TABLA I

\section{CARACTERÍSTICAS DE LA PO BLACIÓ N M UESTRAL}

\begin{tabular}{lcccc}
\hline $\mathrm{N}=117$ & $\begin{array}{c}\text { Número } \\
\text { de mujeres }\end{array}$ & $\begin{array}{c}\text { Porcentaje } \\
\text { de mujeres }\end{array}$ & $\begin{array}{c}\text { Número } \\
\text { de varones }\end{array}$ & $\begin{array}{c}\text { Porcentaje } \\
\text { de varones }\end{array}$ \\
\hline $\begin{array}{l}\text { Población total } \\
\begin{array}{l}\text { Población alto } \\
\text { riesgo(IST>45\%) }\end{array}\end{array}$ & 57 & $48,7 \%$ & 60 & $51,3 \%$ \\
$\begin{array}{l}\text { Población bajo } \\
\text { riesgo(IST<45\%) }\end{array}$ & 52 & $4,3 \%$ & 11 & $9,4 \%$ \\
\hline
\end{tabular}

La proporción de la población de alto riesgo o cribado positivo fue de $13,68 \%$ (16 vs 101) con predominio de varones $69 \%$ (11 vs 5), menor edad media que los de bajo riesgo (60 vs 71 años) y mayor ferritina (477 vs 236 microgramos/litro) (Tabla II). Las relaciones entre las cifras de ferritina, con una media de 268,40 microgramos/litro, y la edad de la población de alto riesgo con la población total de la muestra resultan estadísticamente significativas $(\mathrm{p}<0,05)$, como se refleja en al tabla II. Sin embargo, la relación entre la variable sexo y la población de alto riesgo o cribado positivo resultó estadísticamente no significativa ( $\mathrm{p}=0,123$; chi-cuadrado de Pearson).

Todos los pacientes de la población con cribado positivo, dadas las características muestrales, presentaban enfermedades asociadas (Tabla III). Once casos (69\%) presentaban enfermedades relacionadas con sobrecarga de hierro (5). Destacan por su mayor frecuencia la diabetes mellitus (7 casos) y hepatopatía crónica (6 casos). En cinco pacientes (31\%) se observó la asociación de hepatopatía crónica (virus de la hepatitis C, etíli-

\section{TABLA ॥}

RELACIÓN ENTRE POBLACIÓN DE ALTO Y BAJO RIESGO CON LAS VARIABLES EDAD Y FERRITINA (U DE M ANN-W HITNEY)

\begin{tabular}{lccc}
\hline $\mathrm{N}=117$ & $\begin{array}{c}\text { Población de alto } \\
\text { riesgo (IST }>45 \%)\end{array}$ & $\begin{array}{c}\text { Población de bajo } \\
\text { riesgo (IST }<45 \%)\end{array}$ & $\begin{array}{c}\mathrm{U} \text { de M ann- } \\
\text { Whitney }\end{array}$ \\
\hline Número de paciente & 16 & 101 & - \\
Porcentaje del total & $13,68 \%$ & $86,32 \%$ & - \\
Edad media & $60,8 \pm 20,17$ años & $71,3 \pm 13,7$ años & $P=0,042$ \\
Ferritina media & 477,20 & 236,80 & $P=0,003$ \\
\hline
\end{tabular}

TABLA III

ENFERM EDADES ASO CIADAS CON LA POBLACIÓ N DE ALTO RIESGO

\begin{tabular}{lcc}
\hline $\mathrm{N}=16$ & Número de pacientes & porcentaje \\
\hline Hepatopatía crónica & 6 & $37,5 \%$ \\
Diabetes (DM ID,DM NID) & 7 & $43,75 \%$ \\
Etilismo crónico (>40 g/d) & 4 & $25 \%$ \\
Anemia sideroblástica & 2 & $12,5 \%$ \\
Desnutrición & 3 & $18,75 \%$ \\
\hline
\end{tabular}


ca o no filiada) y diabetes mellitus (insulin-dependiente y no insulin-dependiente). Otras enfermedades asociadas fueron la enfermedad de Paget (1 caso), colangiocarcinoma y cáncer prostático (1 caso) y púrpura trombopénica idiopática (1 caso). Sólo 5 pacientes con sobrecarga de hierro no presentaban ninguna enfermedad asociada con aquella (5).

De los 16 casos positivos, 3 pacientes padecían una desnutrición o hipoproteinemia importante con una transferrina media de $84 \pm 42,46 \mathrm{mg} / \mathrm{dl}$ y mediana de 61 (Tabla IV) que justificaban un IST elevado.

\section{TABLA IV}

CASOS DE DESNUTRICIÓN EN POBLACIÓN DE ALTO RIESGO

Desnutrición IST Sideremia Transferrina Ferritina Edad

$3 / 16$

\begin{tabular}{lccccc}
\hline Caso 1 & $54 \%$ & 40 & 58 & 127 & 83 años \\
Caso 2 & $50 \%$ & 84 & 133 & 96 & 81 años \\
Caso 3 & $54 \%$ & 42 & 61 & 352 & 68 años \\
\hline
\end{tabular}

\section{DISCUSIÓN}

El 13,68\% de los pacientes hospitalizados (16 sobre un total de 117) tuvieron un cribado fenotípico positivo lo que significa que tienen un riesgo elevado de enfermedad por sobrecarga de hierro que es preciso diagnosticar, prevenir y tratar (12). El diagnóstico diferencial de las enfermedades de sobrecarga de hierro exige, por sus implicaciones terapéuticas, la distinción entre hemocromatosis y hemosiderosis (13) por lo que en estos pacientes, previo consentimiento informado, se completa la intervención mediante estudio genético (14) y valoración posterior ambulatoria tanto del probando como de los familiares en aplicación de protocolos de actuación (15).

El grupo con cribado positivo se caracteriza por predominio de varones, ser más jóvenes y tener la ferritina más elevada que el grupo de bajo riesgo lo que define un perfil de paciente con posible mayor afectación y gravedad que se puede beneficiar de un diagnóstico precoz. En este grupo, once pacientes $(69 \%)$ presentaban enfermedades relacionadas con la sobrecarga de hierro (5) frente a cinco pacientes (31\%) considerados probandos con sobrecarga idiopática (16).

La búsqueda activa de casos (11) resulta ser un método adecuado para la detección de casos incidentes de síndromes de sobrecarga de hierro en pacientes hospitalizados $(2,5)$. Además este estudio permite la orientación diagnóstica hacia otros procesos clínicos relacionados frecuentes en este grupo muestral (5) que también se pueden beneficiar de la aplicación de medidas preventivas y terapéuticas e influir en el desarrollo de la morbididad y mortalidad $(17,18)$.

El cribado fenotípico de las enfermedades de sobrecarga de hierro con la determinación del IST resulta efectivo en la práctica clínica por su sensibilidad, especificidad y valor predictivo positivo $(3,19,20)$ por lo que proponemos la inclusión del IST en las determinaciones analíticas básicas a realizar en los pacientes hospitalizados.

\section{AGRADECIMIENTO}

Este trabajo ha sido realizado en parte gracias a una Beca de Colaboración del Ministerio de Educación, Cultura y Deporte.

Los autores de este trabajo agradecen a la doctora Dolores Vigil, del Servicio de Medicina Preventiva del Hospital General Universitario Gregorio Marañón, su ayuda en el análisis de los resultados.

\section{Bibliografía}

1. Powell LW, Subramaniam VN, Yapp TR. Haemochromatosis in the new millennium. J Hepatol 1999; 32 (Supl. 1): 48-62.

2. EASL International Consensus Conference on Haemochromatosis. J Hepatol 2000; 33: 485-504.

3. Olynyk JK, Cullen DJ, Aquila S, Summerville L, Powell LW. A population based study of the clinical expression of the hemochromatosis gene. N Engl J Med 1999; 341: 718-24.

4. Pérez-Aguilar F. Hemocromatosis hereditaria: consideraciones fisiopatológicas, clínicas y terapéuticas. Med Clin (Barc) 2002; 118 (3):103-10.

5. Del Castillo A, López-Herce JA, De Portugal J. Hemocromatosis hereditaria.-Diagnostico clínico: manifestaciones precoces, procesos relacionados y formas atípicas. An Med Interna (Madrid) 2002; 19: 251-6.

6. Edwards CQ, Kushner JP. Screening for Hemochromatosis. N Engl J Med 1993; 328: 1616-20.

7. Bacon BR, Tavill AS. Hemochromatosis and the iron overload syndromes. En: Zakin D, Boyer TD, editores. Hepatology. A textbook of liver disease ( $3^{\mathrm{a}}$ ed.). Philadelphia: Saunders, 1996. p. 1439-72.

8. McLaren CE, McLachlan J, Hallyday JW, Webb SI, Leggett BA, Jazwinska EC, et al. Distribution of transferrin saturation in Australian population: relevance to the early diagnosis of hemochromatosis. Gastroenterology 1998; 114: 543-9.

9. Olynyk JK. Hereditary haemochromatosis: diagnosis and manegement in the gene era. Liver 1999; 19: 73-80.

10. McDonnell SM, Witte DL, Cogswell ME, McIntyre R. Strategies to increase detection of hemochromatosis. Ann Intern Med 1998; 129: 987-92.

11. Wald N, Morris JK. What is a case-finding? J Med Screen 1996; 3: 1.

12. Remacha AF, Carrasco M, Sardá MP, Barceló MJ, Blesa I, Baiget M.
Screening for iron overload and HFE mutations in a university hospital. Haematologica 2000; 85: 873-4.

13. Allgayer H, Romen W. Hemochromatosis or hemosiderosis? Initial misinterpretation of clinical symptoms and laboratory findings in a 62 year-old patient. Z Gastroenterol 2002; 40: 249-54.

14. Zúñiga A, Orera MA. Genética de las sobrecargas férricas. An Med Interna (Madrid) 2002; 19: 195-201.

15. British Committee for Standards in Haematology. Guidelines on diagnosis and therapy: Genetic Haemochromatosis. Darwin Medical Communications. Oxfordshire 2000.

16. Barosi G, Salvaneschi L, Grasso M, Martinetti M, Marchetti M, Bodini U, et al. High prevalence of a screening-detected, HFE-unrelated, mild idiopathic iron overload in Northern Italy. Haematologica 2002; 87: 472-8.

17. De Portugal Álvarez J. Hemocromatosis: del fenotipo al genotipo. An Med Interna (Madrid) 2002; 19: 163-5.

18. Wojcik JP, Speechley MR, Kertesz AE, Chakrabarti S, Adams PC Natural history of C282Y homozygotes for hemochromatosis. Can J Gastroenterol 2002; 16: 297-302.

19. Witte DL. Screening test for hemochromatosis. En: Barton JC, Edwards CQ editores. Hemochromatosis. Cambridge University Press 2000; 519-24.

20. Capuccio J, Phatak PD. Cost-effectiveness of screening for hemochromatosis. En: Barton JC, Edwards CQ, editors. Hemochromatosis. Cambridge University Press 2000; 525-32.

21. Khosravi P, Caño M, Calvo E, Del Castillo A, Portugal J. Cribado fenotípico de hemocromatosis en medicina interna. III Congreso de Investigación de Pregrado en Ciencias de la Salud. Universidad Complutense de Madrid. Madrid 2002 